\title{
New class of quantum echoes motivated by experiments with trapped atoms
}

\author{
Arseni Goussev ${ }^{1}$ and Philippe Jacquod ${ }^{2}$ \\ ${ }^{1}$ School of Mathematics, University of Bristol, UK \\ ${ }^{2}$ Physics Department, University of Arizona, USA
}




\title{
New class of quantum echoes motivated by experiments with trapped atoms
}

\author{
Arseni Goussev ${ }^{1}$ and Philippe Jacquod ${ }^{2}$ \\ ${ }^{1}$ School of Mathematics, University of Bristol, UK \\ ${ }^{2}$ Physics Department, University of Arizona, USA
}

Cargèse, 12-16 July 2010

e-print: arXiv:1006.0339 


\section{Stability of quantum dynamics: Loschmidt echo}

\section{Loschmidt echo ${ }^{1}$}

$M_{\mathrm{L}}(t)=\left|m_{\mathrm{L}}(t)\right|^{2}$ with $m_{\mathrm{L}}(t)=\left\langle\psi\left|e^{i H_{2} t} e^{-i H_{1} t}\right| \psi\right\rangle$

measures separation between $e^{-i H_{1} t}|\psi\rangle$ and $e^{-i H_{2} t}|\psi\rangle$ obtained from an initial state $|\psi\rangle$ in a course of evolution through time $t$ under different Hamiltonian operators $H_{1}$ and $H_{2}$.

\section{Brief background:}

- short times: $M_{\mathrm{L}}(t) \simeq 1-\left(\sigma_{\mathrm{L}} t\right)^{2}$, where $\sigma_{\mathrm{L}}^{2}=\left\langle\psi\left|\Sigma_{\mathrm{L}}^{2}\right| \psi\right\rangle-\left\langle\psi\left|\Sigma_{\mathrm{L}}\right| \psi\right\rangle^{2}$ and $\Sigma_{\mathrm{L}}=H_{1}-H_{2}$

- intermediate times: $M_{\mathrm{L}}(t) \simeq e^{-t \min [\Gamma, \lambda]}$, where $\Gamma=$ width of LDOS and $\lambda=$ Lyapunov exponent

- long times: saturation at $M_{\mathrm{L}, \infty} \simeq \frac{1}{N}$, where $N=$ effective size of Hilbert space

${ }^{1}$ Review articles:

Gorin et al., Phys. Rep. (2006);

Jacquod and Petitjean, Adv. Phys. (2009). 


\section{Echo spectroscopy of trapped atoms}

Consider a cloud of optically trapped "two-level" atoms.

\section{Ramsey spectroscopy:}

- initial state: $|1\rangle|\psi\rangle$

- $\pi / 2$-pulse: $\frac{1}{\sqrt{2}}(|1\rangle+i|2\rangle)|\psi\rangle$

- t-evolution: $\frac{1}{\sqrt{2}}|1\rangle e^{-i H_{1} t}|\psi\rangle+\frac{i}{\sqrt{2}}|2\rangle e^{-i\left(H_{2}+\Delta\right) t}|\psi\rangle$

- $\pi / 2$-pulse: $\frac{1}{2}|1\rangle\left(e^{-i H_{1} t}-e^{-i\left(H_{2}+\Delta\right) t}\right)|\psi\rangle$

$$
+\frac{i}{2}|2\rangle\left(e^{-i H_{1} t}+e^{-i\left(H_{2}+\Delta\right) t}\right)|\psi\rangle
$$

- state $|2\rangle$ population: $\frac{1}{2}+\frac{1}{2} \operatorname{Re}\left\langle\psi\left|e^{i H_{2} t} e^{-i H_{1} t}\right| \psi\right\rangle e^{i \Delta t}$ Interference term is only observed if $|\psi\rangle$ is a pure state.

\section{Modified Ramsey spectroscopy: 2}

- initial state: $|1\rangle|\psi\rangle$

$\rightarrow \pi / 2$-pulse $\rightarrow t / 2 \rightarrow \pi$-pulse $\rightarrow t / 2 \rightarrow \pi / 2$-pulse

- state $|2\rangle$ population: $\frac{1}{2}-\frac{1}{2} \operatorname{Re}\left\langle\psi\left|e^{i H_{2} t / 2} e^{i H_{1} t / 2} e^{-i H_{2} t / 2} e^{-i H_{1} t / 2}\right| \psi\right\rangle$ Interference term is observed even if $|\psi\rangle$ is a thermal mixture.

${ }^{2}$ Davidson's group, PRL (2003); PRA (2004); PRL (2006). 


\section{Echo of new type}

\section{Davidson echo:}

$M_{\mathrm{Da}}(t)=\left|m_{\mathrm{Da}}(t)\right|^{2}$

with $m_{\mathrm{Da}}(t)=\left\langle\psi\left|e^{i H_{2} t / 2} e^{i H_{1} t / 2} e^{-i H_{2} t / 2} e^{-i H_{1} t / 2}\right| \psi\right\rangle$

\section{Different from the Loschmidt echo?}

\section{Yes:}

- short times: $M_{\mathrm{Da}}(t) \simeq 1-\left(\sigma_{\mathrm{Da}} t\right)^{4}$, where $\sigma_{\mathrm{Da}}^{4}=\left\langle\psi\left|\Sigma_{\mathrm{Da}}^{2}\right| \psi\right\rangle-\left\langle\psi\left|\Sigma_{\mathrm{Da}}\right| \psi\right\rangle^{2}$ and $\Sigma_{\mathrm{Da}}=\frac{i}{4}\left[H_{1}, H_{2}\right]$

- intermediate times: $M_{\mathrm{Da}}(t) \simeq e^{-t \min [\Gamma, \lambda]}$

- long times: saturation at $M_{\mathrm{Da}, \infty} \simeq \max \left[\left(\frac{\Delta}{\pi \Gamma}\right)^{2}, \frac{1}{N}\right]$ for $\Gamma \gg \Delta$, where $\Delta=$ mean energy level spacing 


\section{Loschmidt echo vs. Davidson echo}

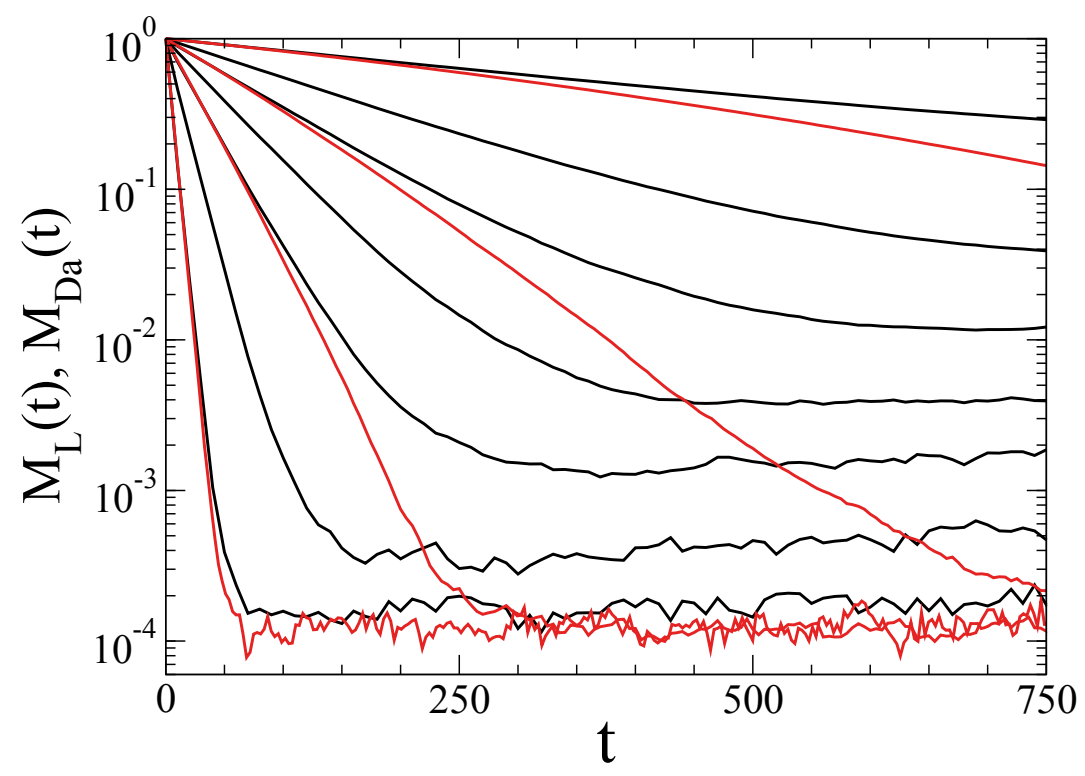

Loschmidt and Davidson echoes for the kicked rotator model

$$
H_{1,2}=\frac{\hat{p}^{2}}{2}+K_{1,2} \cos \hat{x} \sum_{n} \delta(t-n \tau)
$$

with $K_{1}=57, N=8192$, and $K_{2}-K_{1}=5 \cdot 10^{-5}, 1.2 \cdot 10^{-4}, 2.1 \cdot 10^{-4}$ and $5 \cdot 10^{-4}\left(M_{\mathrm{L}}\right.$, red lines from top to bottom $)$, and $K_{2}-K_{1}=5 \cdot 10^{-5}, 9 \cdot 10^{-5}$, $1.2 \cdot 10^{-4}, 1.6 \cdot 10^{-4}, 2.1 \cdot 10^{-4}, 3.1 \cdot 10^{-4}$, and $5 \cdot 10^{-4}\left(M_{\mathrm{Da}}\right.$, black lines, from top to bottom). Curves are averages over 500 initial states. 


\section{Saturation of the Davidson echo}

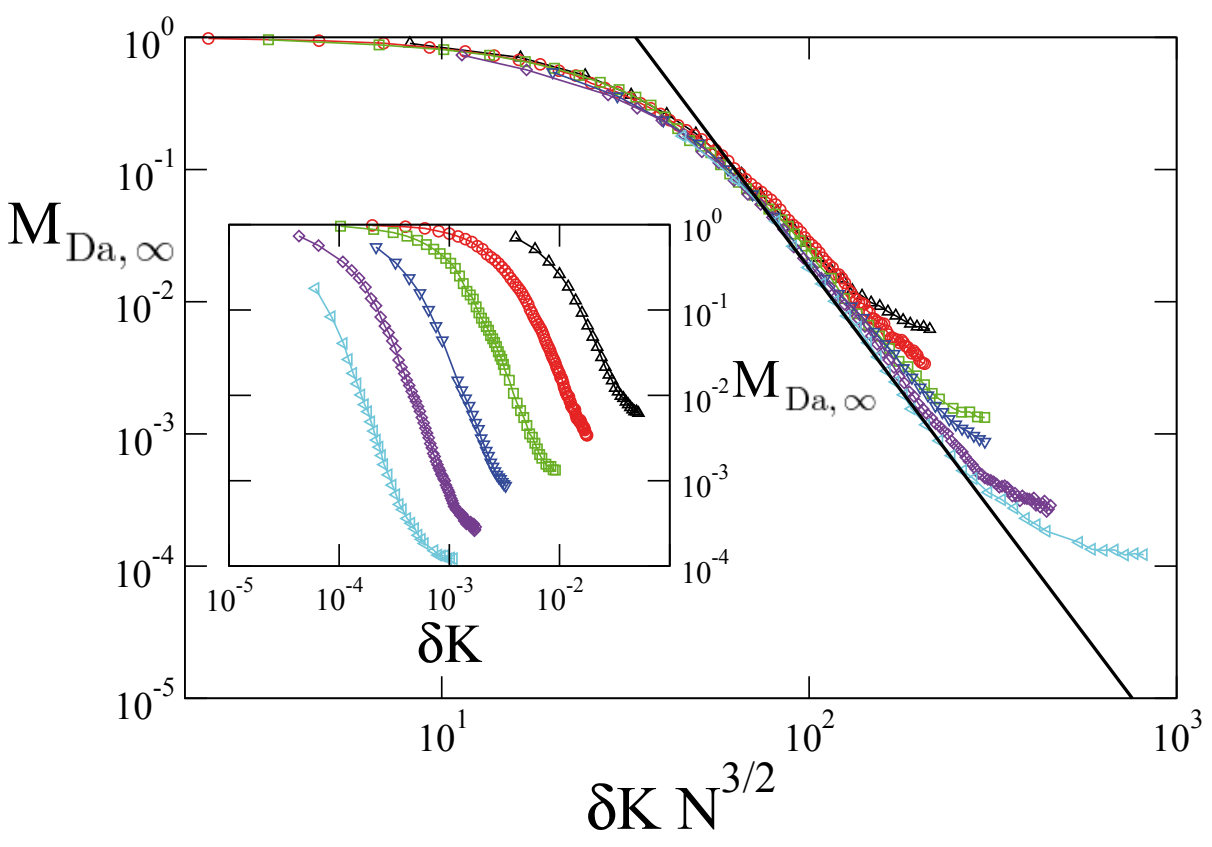

Long-time saturation value of the Davidson echo

$$
M_{\mathrm{Da}, \infty} \sim\left(\delta K^{2} N^{3}\right)^{-2}
$$

(since $\Gamma \propto \delta K^{2} N^{2}$ ) for $K_{1}=57$ and $N=256$ (black), 512 (red), 1024 (green), 2048 (blue), 4096 (violet) and 8192 (cyan). Main panel: rescaled data confirming the analytical prediction. The straight black line indicates a slope of $\propto 1 / x^{3.8}$. Inset: raw data as function of $\delta K=K_{2}-K_{1}$. 


\section{Conclusions}

- The short-time decay of the Davidson echo is quartic (and not quadratic) in time, and is governed by the commutator (and not the difference) of the unperturbed and perturbed Hamiltonians.

- For not too strong Hamiltonian perturbations, the decay of the Davidson echo freezes at values inversely proportional to the square of the perturbation strength (as defined by the width of the local density of states). This allows to estimate the strength of decoherence processes in systems of cold trapped atoms by fitting the saturation value of the Davidson echo, which is easier and more accurate than fitting the Loschmidt echo decay curves over not precisely defined time intervals. 
Technical details: Intermediate times

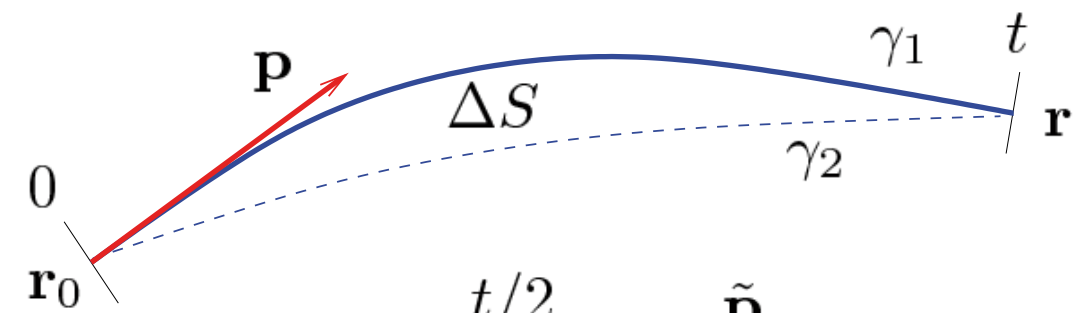

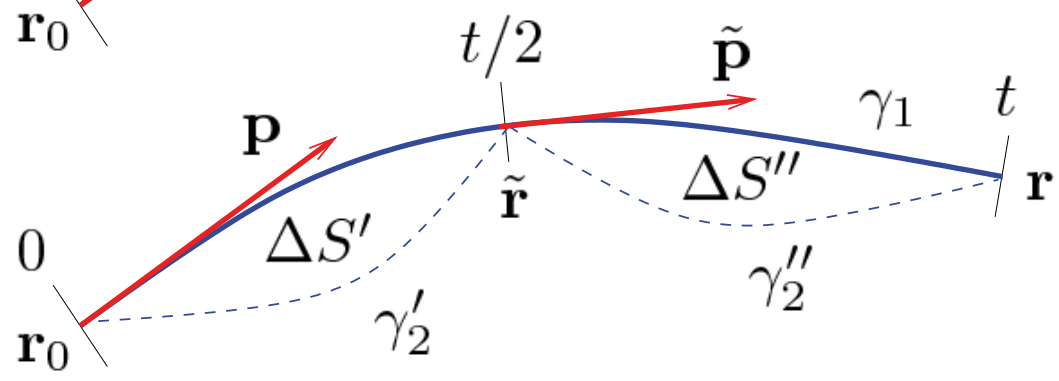

$$
\begin{aligned}
& m_{\mathrm{L}}(t)=(2 \pi)^{-d} \int d \mathbf{p} e^{i \Delta S}|\langle\mathbf{p} \mid \psi\rangle|^{2} \\
& m_{\mathrm{Da}}(t)=(2 \pi)^{-d} \int d \mathbf{p} e^{i\left(\Delta S^{\prime}-\Delta S^{\prime \prime}\right)}|\langle\mathbf{p} \mid \psi\rangle|^{2} \\
& M_{\mathrm{L}, \mathrm{Da}}(t) \simeq{\overline{m_{\mathrm{L}, \mathrm{Da}}(t)}}^{2}+(2 \pi)^{-2 d} \int d \mathbf{p} \int_{\Omega_{\mathbf{p}}} d \mathbf{p}^{\prime}|\langle\mathbf{p} \mid \psi\rangle|^{2}\left|\left\langle\mathbf{p}^{\prime} \mid \psi\right\rangle\right|^{2} \\
& \overline{\exp (i \Delta S)} \simeq \exp \left(-\overline{\Delta S^{2}} / 2\right) \simeq e^{-\Gamma t / 2} \\
& \overline{\exp \left[i\left(\Delta S^{\prime}-\Delta S^{\prime \prime}\right)\right]} \simeq \exp \left[-\left(\overline{\Delta S^{\prime 2}}+\overline{\Delta S^{\prime \prime 2}}\right) / 2\right] \simeq e^{-\Gamma(t / 2+t / 2) / 2}=e^{-\Gamma t / 2}
\end{aligned}
$$




\section{Technical details: Long times}

We first diagonalize the Hamiltonians, $H_{1}=\sum_{u} E_{u}|u\rangle\langle u|$ and $H_{2}=\sum_{v} E_{v}|v\rangle\langle v|$, and expand the initial sate $|\psi\rangle=\sum_{u} c_{u}|u\rangle$.

\section{Loschmidt echo:}

$$
M_{\mathrm{L}, \infty}=\sum_{u, u^{\prime}, u^{\prime \prime}, v} c_{u}^{*} c_{u^{\prime \prime}}\left|c_{u^{\prime}}\right|^{2}\langle u \mid v\rangle\left\langle v \mid u^{\prime}\right\rangle\left\langle u^{\prime} \mid v\right\rangle\left\langle v \mid u^{\prime \prime}\right\rangle
$$

Averaging over an ensemble of random initial states $\left(\overline{c_{u}^{*} c_{u^{\prime}}}=N^{-1} \delta_{u, u^{\prime}}\right)$ yields the saturation $\overline{M_{\mathrm{L}, \infty}}=N^{-1}$.

\section{Davidson echo:}

$$
\overline{m_{\mathrm{Da}, \infty}}=\sum_{u, u^{\prime}, v} \overline{c_{u}^{*} c_{u^{\prime}}}\langle u \mid v\rangle\left\langle v \mid u^{\prime}\right\rangle\left\langle u^{\prime} \mid v\right\rangle\left\langle v \mid u^{\prime}\right\rangle=\frac{1}{N} \sum_{u, v}|\langle u \mid v\rangle|^{4}
$$

Using ${ }^{3}$

$$
|\langle u \mid v\rangle|^{2} \simeq \frac{\Delta}{\pi} \frac{\Gamma / 2}{\left(E_{u}-E_{v}\right)^{2}+(\Gamma / 2)^{2}}
$$

leads to $\overline{m_{\mathrm{Da}, \infty}} \simeq \Delta / \pi \Gamma$, and therefore $\overline{M_{\mathrm{Da}, \infty}}=\left(\frac{\Delta}{\pi \Gamma}\right)^{2}$.

\footnotetext{
${ }^{3}$ Wisniacki et al., PRL (2010) and references within.
} 